\title{
Editora Científica Autônoma e Distribuída sobre Blockchain Privada
}

\author{
Bruno Evaristo $^{1}$, Vagner Nascimento $^{1}$, André Defrémont ${ }^{1}$, Billy Pinheiro ${ }^{1}$, Antônio Abelém ${ }^{1}$ \\ ${ }^{1}$ Grupo de Pesquisa em Redes de Computadores e Comunicação Multimídia (GERCOM) \\ Universidade Federal do Pará (UFPA) \\ Caixa Postal 470 - 66075-110 - Belém - PA - Brasil \\ \{ebec2012, andre.def93\}@gmail.com e \{vagner,billy,abelem\}@ufpa.br
}

\begin{abstract}
The process of publication of scientific articles is made globally by publishers through the Internet and using systems of submission, review and publication. These systems are very expensive, financially as well as computationally, making scientific knowledge restricted to a declining audience. There are some initiatives that try to make the editing process open and aim to promote more democratic access to it. However, in spite of the effort of democratizing the access to scientific production, two challenges are evident: the first is the process of submission, review and publication, and the relationship between authors and publishers is unequal and the second is related to the infrastructure of a publisher who normally responds to the article's publication rights. So, for the sake of giving more governance to the intermediary (editor) over the publication process, we propose an Autonomous and Distributed Scientific Publisher (Distributed Autonomic Scientific Publisher - DASP), an autonomous scientific publisher that works over private(permissioned) blockchain, making it possible for every process to be audited and that ensures the authorial rights to the involved researchers on the publication of each article, lowering the cost for readers with the withdraw of intermediaries (publishers). proof of concept, it was developed a publisher environment with the basic processes for submission and review, to validate the proposal.
\end{abstract}

Resumo. O processo de publicações de artigos global através da Internet utilizando sistemas de submissão, revisão e publicação. Tais sistemas tem um elevado custo financeiro e computacional, tornando o conhecimento científico restrito a um público cada vez menor. Existem iniciativas que tentam tornar o processo de submissão e avaliação abertos e visam promover o acesso mais democráticos a ele. Entretanto, dois desafios ficam evidentes, o primeiro é a relação desigual entre autores e editoras durante os processos de submissão, revisão e publicação; o segundo está relacionado a infraestrutura de uma editora que normalmente responde pelos direitos de publicação do artigo. Neste sentido, visando dar maior governança ao processo de publicação de artigos, nós propomos a Editora Científica Autônoma e Distribuída (Distributed Autonomic Scientific Publisher - DASP), uma editora científica autônoma funcionando sobre uma blockchain privada(permissionada) que permite que todo o processo seja auditado, garantindo os direitos autorais aos pesquisadores envolvidos na publicação de cada artigo e diminuindo o custo para os leitores com a retirada de intermediários. Como prova de conceito foi desenvolvido um ambiente editorial com os processos básicos de submissão e revisão para validar a proposta. 


\section{Introdução}

O método científico permite que as explicações para os fenômenos sejam testados, analisados, discutidos e revisados por pares distintos. Esta validação é realizada através do processo de publicação de artigos, no qual a comunidade científica certifica se os resultados no artigo são suficientes para comprovar as hipóteses levantadas [Job et al. 2009].

Atualmente, o processo de publicações de artigos científicos é realizado de maneira global por grandes editoras como $\operatorname{IEEE}^{1}$ e $\mathrm{ACM}^{2}$ e outras através do uso da Internet e de sistemas de submissão, revisão e publicação como o JEMS ${ }^{3}$ e EDAS ${ }^{4}$. Neles, cada autor envia seus artigos, os quais serão avaliados por revisores externos e, eventualmente publicados de forma impressa ou digital.

Em consonância com a crescente migração para o meio digital, o setor financeiro passou a criar e manipular digitalmente seus ativos, culminando com o aumento no interesse da indústria e da academia pelo desenvolvimento de criptomoedas e de sua tecnologia base, a blockchain. Esta permite que informações sejam armazenadas de forma descentralizadas, com tolerância a falha e auditável [Zheng et al. 2016].

As aplicações que rodam sobre uma rede blockchain são chamadas de Decentralized Applications (Dapps) e são formadas, principalmente, por partes lógicas executadas na Blockchain, denominadas de contratos inteligentes (smart contracts). Estes expressam a lógica de forma confiável e verificável, tornando-se uma boa solução para estabelecer confiança entre entidades distintas [Frantz and Nowostawski 2016].

Neste contexto, para prover serviços de revisões e publicações de artigos científicos de maneira transparente, propomos a Editora Científica Autônoma e Distribuída (Distributed Autonomic Scientific Publisher - DASP). Nela, são consideradas características de editoras conhecidas e seu processo de gestão, com a diferença que na DASP propomos que a própria comunidade gerencie o processo. Assim, temos uma abordagem mais ampla e transparente, onde todas as políticas de consenso estão vinculadas aos contratos inteligentes, garantindo melhor gestão do processo de publicação.

Como contribuições científicas deste artigo, destacamos a adaptação do modelo de Organizações Autônomas Descentralizadas (Decentralized Autonomous Organization - DAO) para o contexto de publicações científicas, usando um esquema de comprometimento não iterativo para troca de chaves criptográficas dos autores e revisores com a DASP, mantendo-os anônimos. A outra contribuição é a infraestrutura fornecida pela DASP aos autores e revisores que permite escalabilidade, confiança, segurança e auditabilidade.

Este trabalho está estruturado da seguinte forma: A Seção 2 introduz as tecnologias empregadas na DASP; A Seção 3 discute os trabalhos relacionados; A Seção 4 descreve a DASP em detalhes; A Seção 5 apresenta a prova de conceito da DASP; e, por fim, a Seção 6 conclui esse trabalho e aponta os trabalhos futuros.

\footnotetext{
${ }^{1}$ https://www.ieee.org/

${ }^{2}$ https://www.acm.org/

${ }^{3}$ https://jems.sbc.org.br/

${ }^{4}$ https://edas.info/
} 


\section{Referencial Teórico}

Nesta seção, apresentamos a base teórica para discutir o processo de gestão por parte das editoras nas publicações de artigos científicos. Assim como, as tecnologias presentes em redes blockchain, que viabilizam a criação de contratos inteligentes e o armazenamento distribuído de arquivos.

\subsection{O processo de Publicação de Artigos}

O processo de publicação de um artigo científico envolve o autor, o revisor, o editor, a editora e o leitor. Cada um desempenha um papel específico no sistema, podendo a mesma pessoa desempenhar diferentes papéis, porém, não concorrentemente [Ferraz and Navas 2016]. A seguir detalharemos as funções exercidas por cada participante em uma editora científica e descreveremos os modelos de revisão comumente usados por elas.

- Autor: Realiza a escrita do artigo e é responsável pela submissão do mesmo. Todo o trabalho científico e até mesmo a diagramação do conteúdo a um formato pré-estabelecido é de sua responsabilidade;

- Revisor: Avalia os artigos e atribue notas que determinam a aceitação do artigo. Não é raro que autores de um determinado períodico sejam convidados a compor o corpo de revisores do mesmo. Tal função é, normalmente, não remunerada, mas de reconhecido prestígo dentro do seu respectivo campo de atuação;

- Editor: Responsável por receber as revisões, organizar os artigos aceitos e gerar a publicação final;

- Editora: Organizam os editores e revisores, fazendo as chamadas para publicações, nas quais os autores submetem seus artigos. Dependendo da organização do evento, os autores e leitores são taxados de alguma forma para realizarem a publicação ou leitura do trabalho;

- Leitor: Acessa os artigos que foram publicados, podendo eventualmente pagar por isso.

Com o avanço das tecnologias de processadores de texto e diagramação, como, por exemplo, ( $\left.\mathrm{LT}_{\mathrm{E}} \mathrm{X}^{5}\right)$, os trabalhos dos editores e das próprias editoras são minimizados, retirando todo o esforço manual do processo de diagramação por parte dos editores, restando a estes a escolha dos revisores e unificação das notas; e às editoras a escolha dos editores.

O processo de publicação é iniciado com a submissão do artigo seguida da distribuição destes para os revisores previamente cadastrados, alguns sistemas já fazem esse trabalho automaticamente, como o JEMS, substituindo o fator humano que pode influenciar no processo. Na sequência, cada revisor recebe uma notificação de que uma revisão foi solicitada podendo aceitar ou negar. Esta dinâmica entre editoras, revisores e autores pode variar já que existem inúmeros modelos de revisões propostos por pesquisadores. Entre os principais modelos, podemos citar:

- Halfblind: Somente os revisores conhecem os nomes dos autores do artigo.

- Fullblind: Nem o autor, nem o revisor sabem a identidade um do outro.

\footnotetext{
${ }^{5}$ https://www.latex-project.org/
} 
- Open peer review: A identidade dos autores e dos revisores são reveladas, tornando conhecido todos os envolvidos no processo;

- Triple-blind: A identidade dos autores, revisores e editores é oculta;

- Crowdsourced peer review: Permite que a comunidade em geral contribua para o processo de revisão;

- Open pre-review manuscripts: Os trabalhos ficam acessíveis (via Internet) antecipadamente, ou em sincronia com qualquer procedimento formal de revisão por pares. Normalmente, esse tipo de revisão é usado em repositórios institucionais.

Um levantamento abordando a avaliação por pares lista a saturação dos revisores em relação aos métodos existentes e alguns vícios de avaliações, já que no meio acadêmico, um grupo de estudo normalmente reconhece o trabalho ou a área de atuação que está envolvida um outro grupo, o que pode acabar gerando vantagens avaliativas comparado com grupos acadêmicos de estudos não tão conhecidos [Mulligan et al. 2012].

\subsection{Blockchain}

A tecnologia blockchain funciona como um livro de registros distribuído, permitindo a troca de recursos digitais que não são emitidos ou controlados por uma autoridade central. Cada transação é assinada com uma chave $\mathrm{PGP}^{6}$, garantindo a autenticidade, e o conjunto de transações são organizadas em blocos ligados por uma árvore binária formada com os hash das últimas transações [Greve et al. 2018]. Desta forma, as informações armazenadas na blockchain são consideradas de alta integridade [Tschorsch and Scheuermann 2016].

São acordos automatizados nos quais a execução é realizada por meio de ambientes de desenvolvimento [Cachin 2016], como Chaincode, no Fabric ${ }^{7}$ e no Ethereum ${ }^{8}$. Esses contratos, nada mais são que condições ou regras criadas pelo próprio usuário. Assim, quando as premissas são alcançadas, uma condição pré-estabelecida (programada) é executado automaticamente.

Os contratos inteligentes são códigos auto-executáveis usados para configurar as redes baseado em blockchain, habilitam as Organizações autônomas descentralizadas (Decentralized Autonomous Organization - DAO), que são organizações de decisões descentralizada, conhecidas também como iniciativas de fundo de centro vazio, onde cada membro da aplicação tem seu poder de votação baseado em regras pré-estabelecidas e flexíveis via contratos inteligente, que rodam de forma autônoma e transparente em uma blockchain [DuPont 2017].

\subsection{InterPlanetary File System (IPFS)}

Fala-se de aplicações descentralizadas e distribuídas, porém é necessário fornecer mecanismo de armazenamento em larga escala. É nesse contexto que o IPFS surge, como uma alternativa de conectar dispositivos computacionais através de um sistema de arquivos, ou seja, um banco de dados distribuído [Benet 2014].

O IPFS cria uma estrutura simples e flexível na rede através de nós conectados, gerando uma árvore de dados com hashes. Após a instalação do IPFS, o usuário gera um

\footnotetext{
${ }^{6}$ https://www.openpgp.org/about/

${ }^{7}$ https://hyperledger-fabric.readthedocs.io/en/release-1.3/chaincode.html

${ }^{8} \mathrm{http}: / /$ www.ethdocs.org/en/latest/contracts-and-transactions/contracts.html\#what-is-a-contract
} 
primeiro nó, o qual permite ler e interagir com outros hashes, formando assim a árvore de hashes. O IPFS também gera um daemon, acessível via CLI e interface web, onde é possível gerenciar, adicionar e organizar as identidades e suas conexões correspondentes.

\section{Trabalhos Relacionados}

Nesse cenário detalhamos abaixo cada característica citada acima e por fim geramos uma tabela comparativa entre as propostas que contribuem no processo de publicação.

- Gestão de Processo: Essa característica demonstra os mecanismos administrativos que regem o processo editorial, baseado nas pesquisas relacionados aos modelos clássicos do Institute of Electrical and Electronics Engineers (IEEE)[IEEE 2013], Sociedade Brasileira de Computação (SBC)[SBC 2015] e Association for Computing Machinery (ACM) [Shurkin, Joel 2017], fica nítido o modelo de governança centralizada, em contrapartida as propostas de ciência aberta tem como premissa descentralização da gestão, nossa proposta segue essa premissa, onde não se tem uma entidade central administrando o processo.

- Banco de Dados: O armazenamento das informações em um processo editorial tradicional é centralizada em repositórios mantidos pelas editoras. Até mesmo iniciativas visando descentralização, como EUREKA [EurekaPlatform 2018] precisam de um servidor central organizando as informações. Nossa proposta (DASP) tem como premissa o uso de um banco de dados distribuído (IPFS) assim como a proposta Open Science Network (OSN)[Partners 2018]

- Auditoria: Registros de informações de artigos, processos de revisão, e históricos em geral, são extremamente difíceis de serem encontrados nos modelos clássicos, demostrando a ausência de transparência no processo. Nesse ponto, Decentralized Research Platform (DEIP)[DEIP 2018] e a DASP surgem com a idéia do uso de blockchain como mecanismo de registro público, uma vez que partimos da idéia que qualquer membro pertencente a comunidade que fará uso da solução, poderá ter acesso a qualquer tipo de informação de histórico em geral, fornecendo transparência e confiança entre os entes envolvidos no processo.

- Direitos Autorais: Normalmente os lucros e dividendos, majoritariamente, ficam com as editoras por conta da organização e gestão do processo. Na contramão desse cenário, EUREKA, OSN e DEIP fornecem mecanismos próprios de taxação, porém usando a rede Ethereum $^{9}$, que é uma rede pública, sem um atrelamento direto entre os entes virtuais e os entes físicos, dificultando a segurança. Tendo isso em vista, na DASP o autor tem o poder de decidir sobre o futuro da sua produção, onde pode deixar o acesso totalmente público ou cobrando alguma taxa mínima, através de uma blockchain permissionada, sem intermediários (editora). A ideia é ser justo com quem mais trabalha em todo processo de submissão e revisão.

- Inovação: Observamos que os sistemas editoriais tradicionais apresentam dificuldade em expor transparência. Partindo da ideia de descentralização na governança e nos mecanismos estruturais, EUREKA, DEIP e OSN surgem com o princípio de inovar os mecanismos de publicação, trazendo a possibilidade de classificação de pesquisas entre os cientistas, além de focar na facilitação da disseminação de trabalhos acadêmicos. Visando o cenário inovador dessas propostas, a DASP surge

\footnotetext{
${ }^{9}$ https://www.ethereum.org/
} 
com a idéia de humanizar ainda mais o processo, com a idéia de aproximar o autor do revisor, possibilitando o compartilhamento de discussões sobre as revisões através do uso de revisões secundárias, além disso a DASP, fornece a possibilidade de gerar instâncias da aplicação, permitindo criar processos editoriais de comunidades específicas ao instanciar a DASP.

\begin{tabular}{|l|l|l|l|l|l|l|l|}
\hline Características & \multicolumn{3}{|c|}{ Sistemas Editoriais Clássicos } & \multicolumn{3}{c|}{ Propostas de Ciência Aberta (Blockchain) } \\
\hline & IEEE & ACM & SBC & EUREKA & DEIP & OSN & DASP \\
\hline Gestão de Processo & Centr. & Centr. & Centr. & Descen. & Descen. & Descen. & Descen. \\
\hline Banco de Dados & Centr. & Centr. & Centr. & Centr. & Descen. & Descen. & Descen. \\
\hline Auditória & Não & Não & Não & Sim & Sim & Sim & Sim \\
\hline Direitos Autorais & Desigual & Desigual & Desigual & Desigual & Desigual & Desigual & Igualitário \\
\hline Inovação & Nada Relac. & Nada Relac. & Nada Relac. & Descen. & Descen. & Descen. & Descen. \\
\hline
\end{tabular}

Tabela 1. Comparação das propostas.

A Tabela 1 apresenta uma comparação das características apresentadas anteriormente nesta seção, mostrando os ambientes computacionais voltados à governança de sistemas editoriais clássicos e as propostas de editoras que visam inovar no processo de governança, tomando como base a transparência, descentralização do processo e uma relação mais justa entre os atores envolvidos.

É possível identificar que as propostas centralizadas causam um desequilíbrio, com custo alto para leitores e as organizações que acessam seus repositórios. Em contrapartida, várias propostas surgiram, como, por exemplo, o EUREKA o qual seria uma aplicação de revisão de artigos ligada a plataforma de submissão ScienceMatters - Já o DEIP fornece apoio em pesquisas iniciais, através de angariação de recursos e da transformação de projetos de pesquisa em ativos digitais contáveis via tokenização. $\mathrm{O}$ OSN é uma rede pública (ethereum) de pesquisa distribuída que usa a tecnologia blockchain para uma transmissão transparente de informações de valor e um realinhamento significativo de incentivos.

Neste contexto, a DASP, além de gerir todo um processo editorial, é capaz de fornecer plataformas de divulgação de artigos acadêmicos, usando uma blockchain permissionada, ainda não explorada pelas novas soluções, relacionando mecanismos de taxação igualitária entre os entes envolvidos no processo e fomentando a relação entre a comunidade visando as variadas áreas que as constituem.

\section{Editora Científica Autônoma e Distribuída (DASP)}

Visando essas novas propostas de gestão, a DASP surge como uma alternativa para aperfeiçoar a governança no processo de publicação, promovendo a disrupção ao aplicar gerenciamento distríbuído e autônomo entre as entidades envolvidas em todo o processo. Para isso, a proposta utiliza como base o conceito das DAO. Os envolvidos mantêm os papéis de revisor e autor, porém tudo controlado pela DASP, sem intervenção humana, possibilitando o próprio autor tenha alguma recompensa com a publicação, seja financeiro ou através de algum outro bônus que poderá ser decidido entre a comunidade envolvida.

Ao instanciar a DASP, os revisores serão convidados, depois os próprios autores que tiverem artigos aceitos podem virar revisores. Os membros da comunidade podem dar notas para as revisões, assim aumenta ou diminui a probabilidade daquele revisor 
receber outro artigo para revisar, funcionando como um método de retroalimentação na comunidade. Além de todos esses mecanismos, podemos avaliar a não revisão de um artigo aceito por um revisor, o que lhe acarretaria uma nota baixa na plataforma.

A DASP organiza as relações entre os atores da comunidade através de contratos inteligentes executados na rede. A partir deles os membros da comunidade podem assumir o papel de autor, leitor, revisor e revisor dos revisores. Tudo vai depender do quanto a comunidade acredita no individuo para desempenhar um papel específico. Sendo assim, a ideia central da DASP é aumentar a relevância da comunidade e não apenas criar um software, entendemos que o software é importante para prova de conceito neste artigo, mas estamos propondo um modelo de relação que prestigie a comunidade e não as editoras.

\subsection{Arquitetura}

A Figura 1 mostra a arquitetura de componentes da DASP usando o padrão de projeto Model-View-Controller (MVC) [de Lemos et al. 2013]. Na camada modelo estão presentes os módulos que irão se comunicar com a tecnologia de rede (Hyperledger) e armazenamento (IPFS) que serão utilizados. A camada de comunicação é composta pelos gerenciadores(usuário) usando notações de objetos em JavaScript e HyperText Markup Language (HTML). Na camada controle estão as partes relacionadas as funcionalidades responsáveis pela gerência da DASP, sendo ela dividida em quatro componentes que são apresentados a seguir:

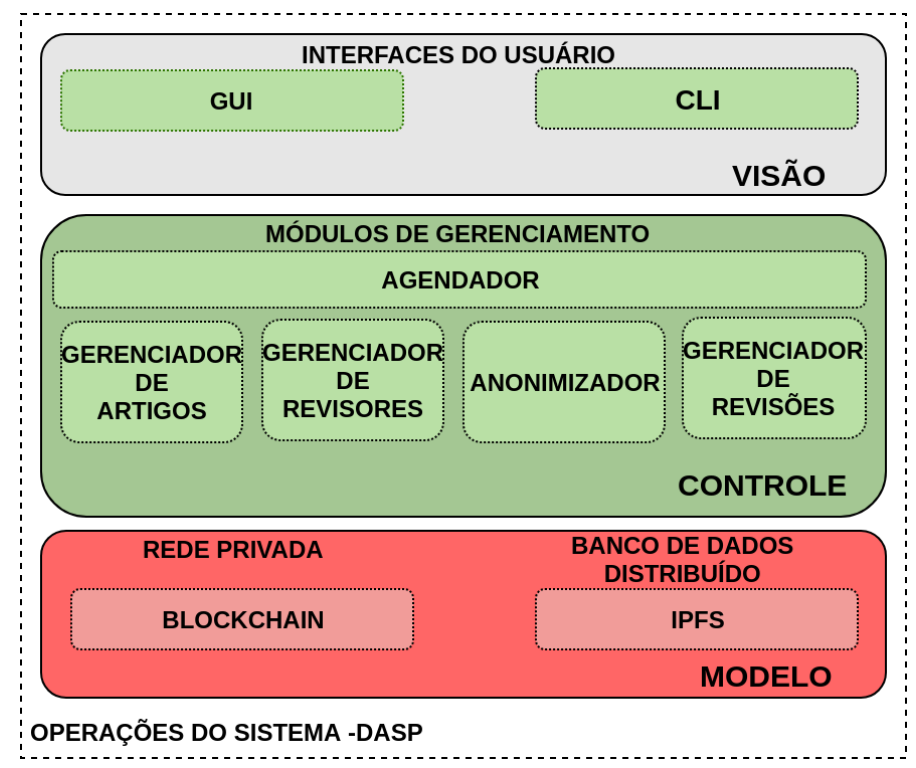

Figura 1. Arquitetura da DASP

- Agendador: Responsável pela definição de tempo e gerenciamento da comunicação entre os demais módulos.

- Gerenciador de Artigos: É responsável pela organização de submissão de arquivos e verificar se o documento está sendo enviado para o ente correto.

- Gerenciador de Revisores: É responsável pela escolha de revisores de forma randômica, mas considerando as condições de escolhas do revisor, como a quantidade de pontos ou tokens que o revisor possui e o número de revisões já realizadas. Sendo esta escolha realizada pela aplicação, sem qualquer intermediário. 
- Anonimizador: Quando uma submissão é realizada, um conjunto de chaves temporárias é gerado para o autor. O sistema distribui a solicitação de revisão para os revisores, que compõem a comunidade, esses revisores geram através da própria interface do sistema um novo endereço blockchain, ou seja, uma chave pública e uma chave privada, e este novo endereço que é desconhecido para todos os entes, passa a ser uma outra carteira, só que temporária, que permanecerá enquanto o processo de revisão estiver ativo. Ao final do processo de revisão, a chave do revisor e do autor são associadas as chaves temporárias, anexando o processo de revisão ao histórico do revisor e a submissão é relacionada ao autor. Tal abordagem utiliza o conceito de comprometimento, no qual gera-se um valor secreto que posteriormente é utilizado para comprovar a autoria [Di Crescenzo et al. 2001].

- Gerenciador de Revisão: É a função responsável pela verificação da qualidade da revisão, que será determinada pela própria comunidade. Neste processo encontrase a função lógica avaliativa em conjunto, entre o autor, a revisão em si e a comunidade, o método avaliativo é observado através de discussões do quanto o avaliador foi coerente em suas avaliações, e destina-se tokens ou pontos, que serão uma espécie de pagamento pela avaliação ter sido julgada por todo o conjunto citado anteriormente, como justa e altamente argumentativa.

A Figura 2 apresenta o diagrama de atividades da DASP, mostrando como funciona o processo de submissão e avaliação do artigo usando a aplicação, que para executar estas tarefas, fará uso de contratos inteligentes que serão assinados por cada um dos envolvidos, desta forma a DASP sabe exatamente o que cada ator fez no processo, permitindo a auditoria. Cada um dos atores terão uma chave pública e uma chave privada com a qual serão identificados no sistema, garantindo assim a privacidade e o não repúdio (não é possível dizer que não foi ele que fez determinada ação).

O processo é iniciado quando o autor submete o artigo que é instanciado no IPFS, onde é gerado um Hash, o arquivo então é enviado para a DASP, que está funcionando sobre uma rede privada usando a tecnologia Hyperledger Fabric ${ }^{10}$ baseado no conceito de empresa de centro vazio (DAO), ou seja, não existe uma instituição controlando a aplicação, sendo a gestão realizada pelos entes da comunidade.

A partir desse momento, a DASP recebe o arquivo e realiza a escolha dos revisores, sendo que nessa etapa o revisor sorteado tem a opção de aceitar ou não a revisão do artigo. Na sequência, a DASP entra no período de revisão. Após a submissão das revisões pelos revisores, será gerada a resposta se a revisão foi aceita ou rejeitada. Em caso de aceite, o artigo é publicado e a revisão por escolha do autor é tornada pública ou não. Caso o artigo seja rejeitado, é enviado ao autor a revisão, o qual pode tornar pública ou não a revisão. Se esta for pública, a revisão estará passível de revisão pela comunidade.

\section{Prova de conceito: DASP}

A DASP surge à medida que movimentos voltados a ciência aberta se amadurecem e ganham visibilidade em comparação ao modelo clássico existente, que centraliza e restringe a relação entre os entes que participam da comunidade de pesquisa. Na contramão desse modelo, fazemos uso da blockchain, uma tecnologia que surgiu na indústria com objetivos de prover maior confiança entre os pares, excluindo intermediários, e além disso, propor

\footnotetext{
${ }^{10}$ https://www.hyperledger.org/projects/fabric
} 


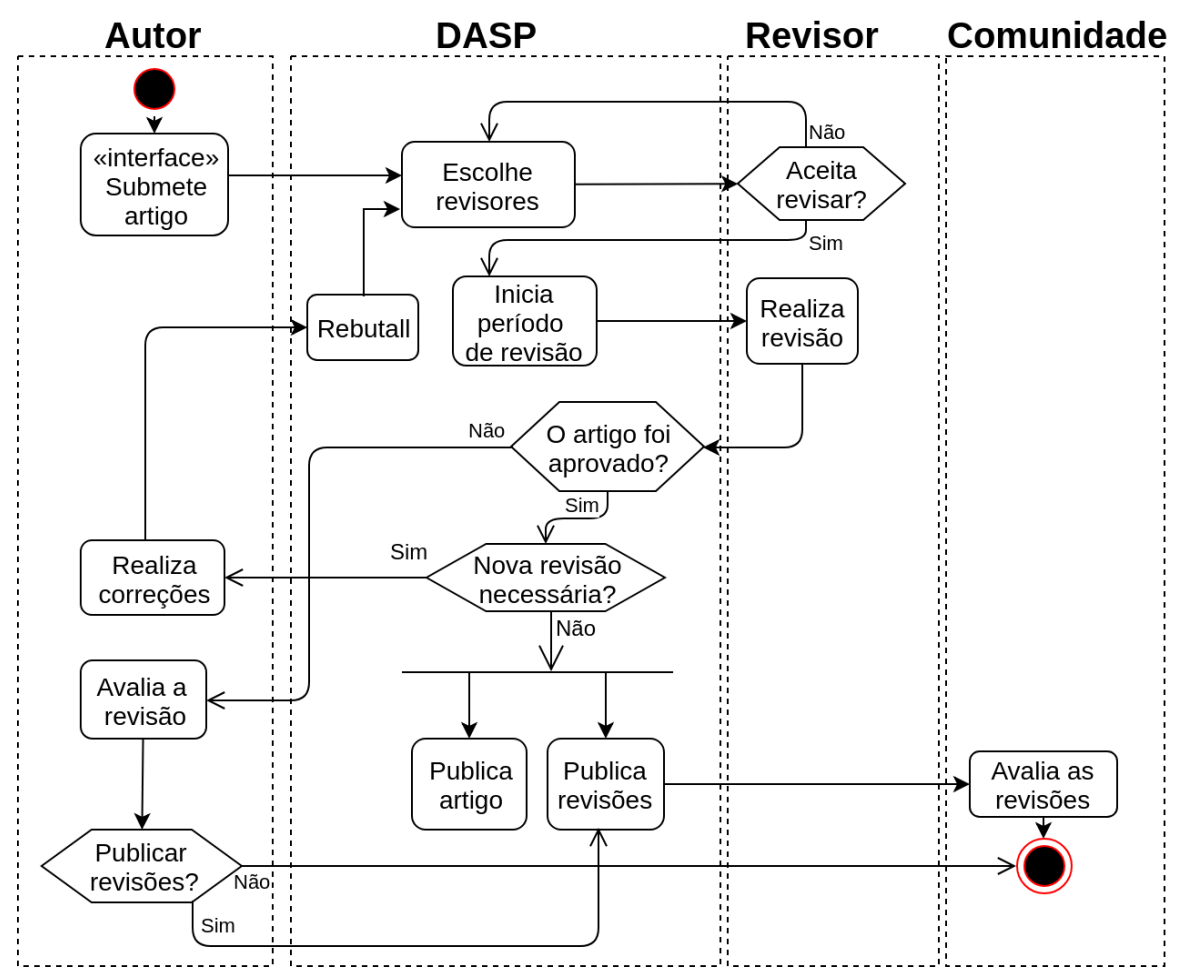

Figura 2. Diagrama de Atividades da DASP

formas de explorar e desenvolver aplicações que possam estabelecer modelos inovadores de relações na comunidade acadêmica.

A Figura 3 apresenta como os diferentes componentes da DASP se comunicam. Com intuito de fundamentalizar o uso do conceito da DAO para a solução, pois este provê um modelo de negócio autônomo e distribuído, no qual a comunidade se auto governa, sem nenhum ente regulador, gerente, ou governo controlando as ações que serão tomadas baseando-se em consenso de regras imutáveis, escritas na blockchain, na forma de contratos inteligentes.

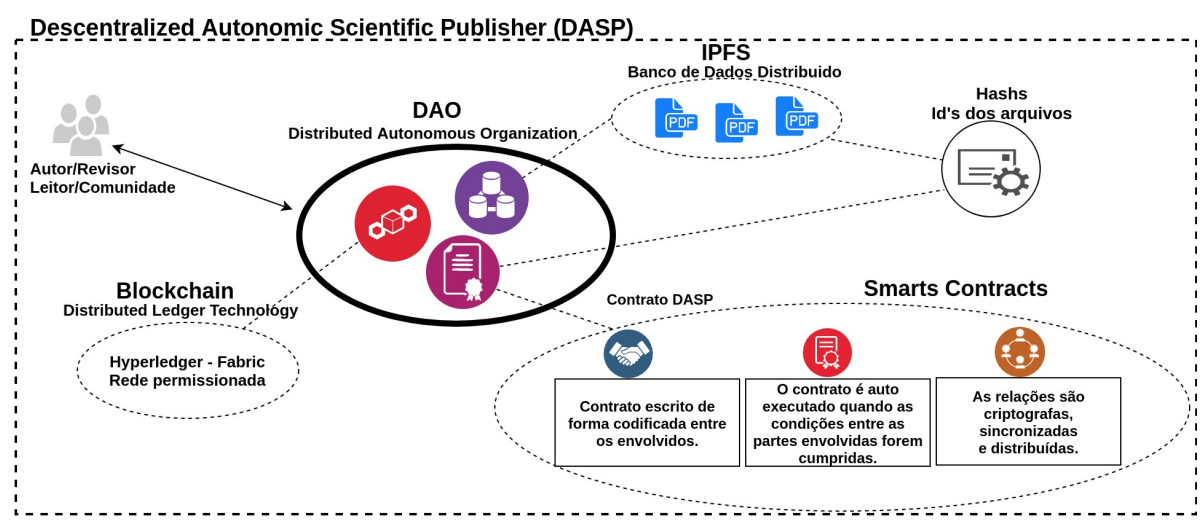

Figura 3. Componentes para Implementação da DASP

A plataforma Hyperledger, como demostrada na Figura 3, é utilizada como base para a criação da DASP. Neles são executados os passos de instruções de envio do arquivo, submissão, revisão do artigo, tempo de publicação e revisão, ou seja, todo o processo que 
envolve os prazos (tempos) e interações entre os entes, junto com as especificações em relação a cada decisão da comunidade, do revisor ou do autor em específico.

Partindo das explicações de funcionamento do sistema, o IPFS tem como função a DASP o armazenamento dos arquivos submetidos, ou seja, quando o autor usa a aplicação (DASP) para enviar um arquivo, esse arquivo é inserido no IPFS, que automaticamente gera um hash que será o identificador daquele arquivo, é através desse identificador (ID) que o revisor irá localizar o arquivo que será visualizado e, posteriormente irá entrar em processo de revisão.

Como na aplicação existe a possibilidade de várias revisões no mesmo trabalho, seja pela escolha primária que é realizada de forma randômica dos revisores ou, através da revisão exposta à comunidade, quando é pedida pelo autor, funciona como uma espécie de segunda opinião, onde é gerado um novo ID para o arquivo, já que o mesmo passou por modificações.

A estrutura da aplicação está disponível publicamente no endereço (https://gitlab.com/gercom/dasp-hyperledger). Para testar a aplicação basta utilizar o ambiente de teste do Hyperledger chamado de Hyperledger Composer Playground que é um ambiente de prototipagem e teste. Através do endereço (https://composerplayground.mybluemix.net/login), efetue o download do GitLab o arquivo de nome "dasp-net@0.0.1.bna"e carregar o arquivo no playground, conforme a Figura 4.

Após a implantação da rede de negócio da DASP no playground, é necessário conectar a aplicação DASP a blockchain, onde será possível visualizar os arquivos e efetuar os testes descritos nesta prova de conceito. A Figura 4, mostra a aplicação DASP pronta para efetuar os testes e criar autor e revisor atráves da DASP(Admin), é nesse momentos que os atores poderão interagir, baseado nas permissões definidas no contrato inteligente.

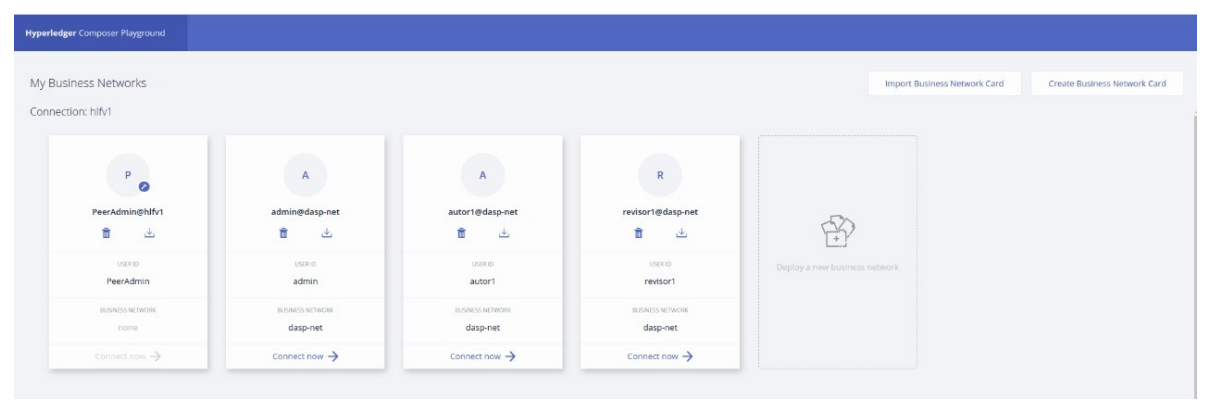

Figura 4. DASP conectada

Após a criação dos atores, é possível iniciar o processo de submissão do artigo por parte do autor, a Figura 5 mostra o registro de submissão dos autores, contituído de detalhes como o tipo de revisão, o hash de identificação do artigo, o ID do autor.

A Figura 6, mostra o registro dos artigos submetidos, como o tipo de revisão, se foi aceito ou não por algum revisor e os IDs de identificação de autor e revisor. Nesse processo, o acesso ao artigo é definido via contrato inteligente, então só os entes autorizados pelo contrato que seguem o fluxo editorial poderão ter acesso ao trabalho para efetuar a revisão, e posteriormente reenviar o resultado de avaliação do trabalho.

A Figura 7, mostra os registro de artigos aceitos pelos revisores, onde cada hash 


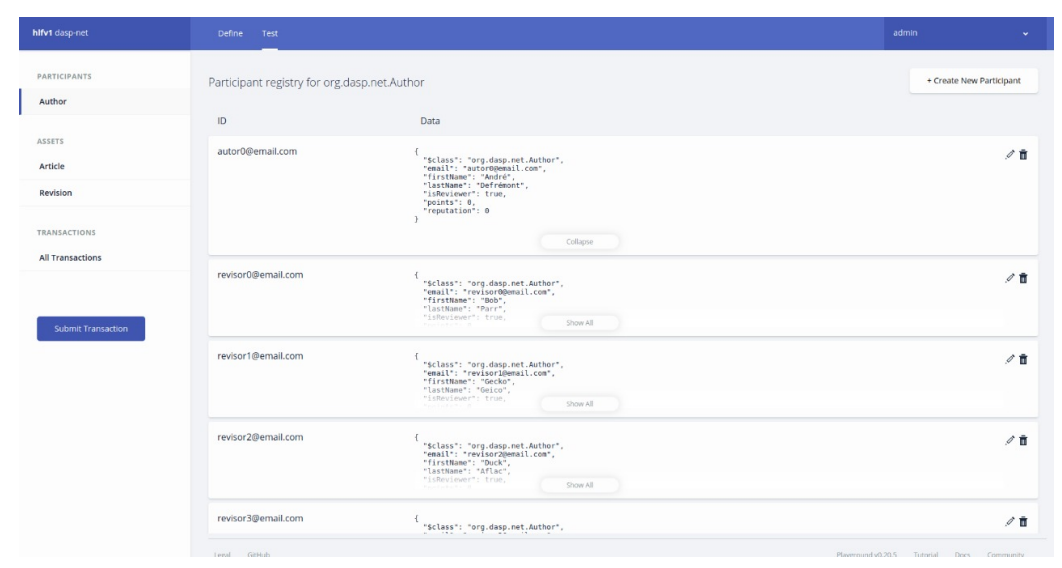

Figura 5. Registro de Submissão dos Autores

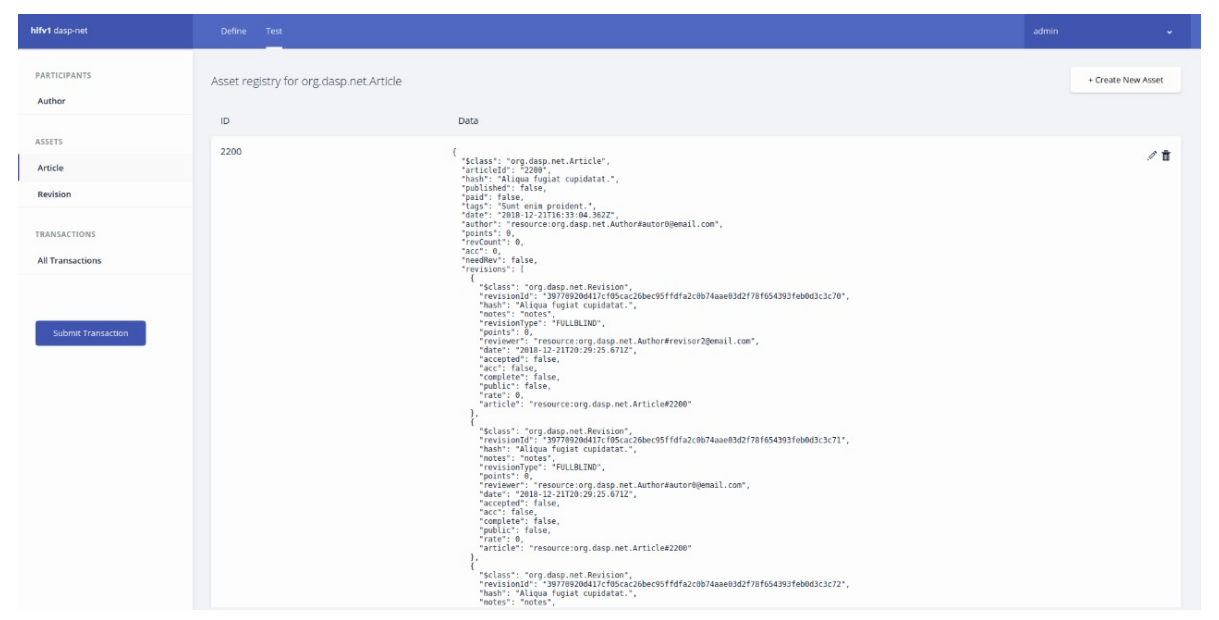

Figura 6. Registro de Artigos Submetidos

de transação e ID do autor fica vinculado ao $I D$ do revisor, e as modificações no artigo ficam registradas na rede blockchain, além do registro das informações como o resultado da avaliação do artigo submetido e as políticas de visualização das revisões (ex: se serão públicas ou privadas a revisão do trabalho à comunidade).

As Figura 8 e 9 mostram as funcionalidades, que seriam os tipos de transações que são usados no processo, como:

- Bootstrap: Gera automaticamente atores e revisores para teste;

- CreateRevision: É respónsavel pela criação de uma nova revisão, é usada quando o autor realizar uma submissão de artigo;

- NewArticle: É usada em caso de uma nova submisão de artigo;

- NewAutor: Quando o autor deseja criar um ID na aplicação;

- NewHash: Ferramenta que gera um novo hash, quando o artigo sofrer algum tipo de modificação, esse mecanismo normalmente é usado quando revisor realiza a avaliação do atrabalho e reenvia o resultado do trabalho, pois toda vez que o trabalho passa por algum tipo de modificação o hash é modificado automaticamente, sem perder o vínculo entre as partes envolvidas;

- PublishRevision: Opção ativada quando a revisão é publicada ao autor; 


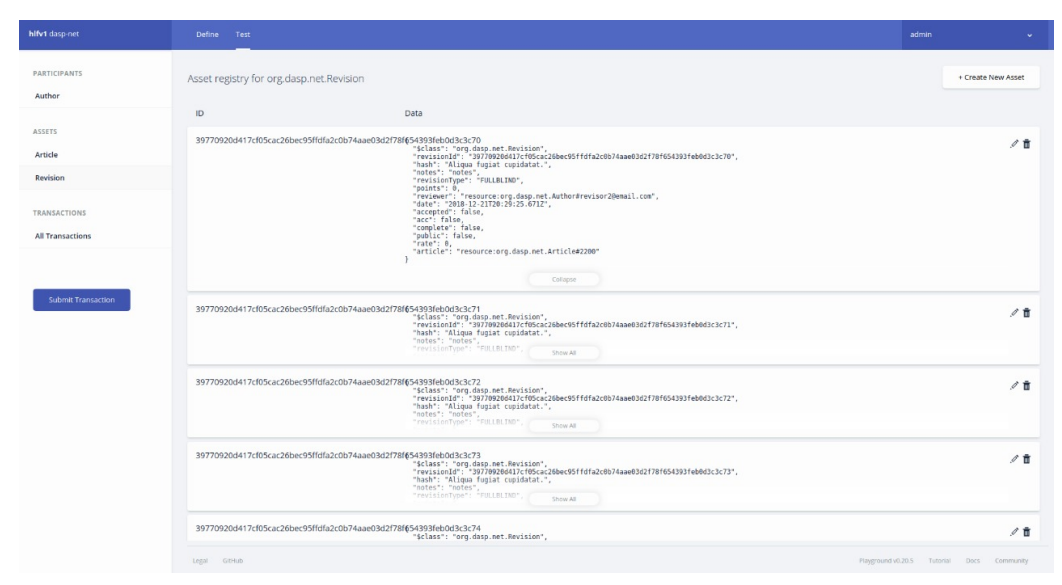

Figura 7. Registro de Trabalhos Aceitos pelos Revisores

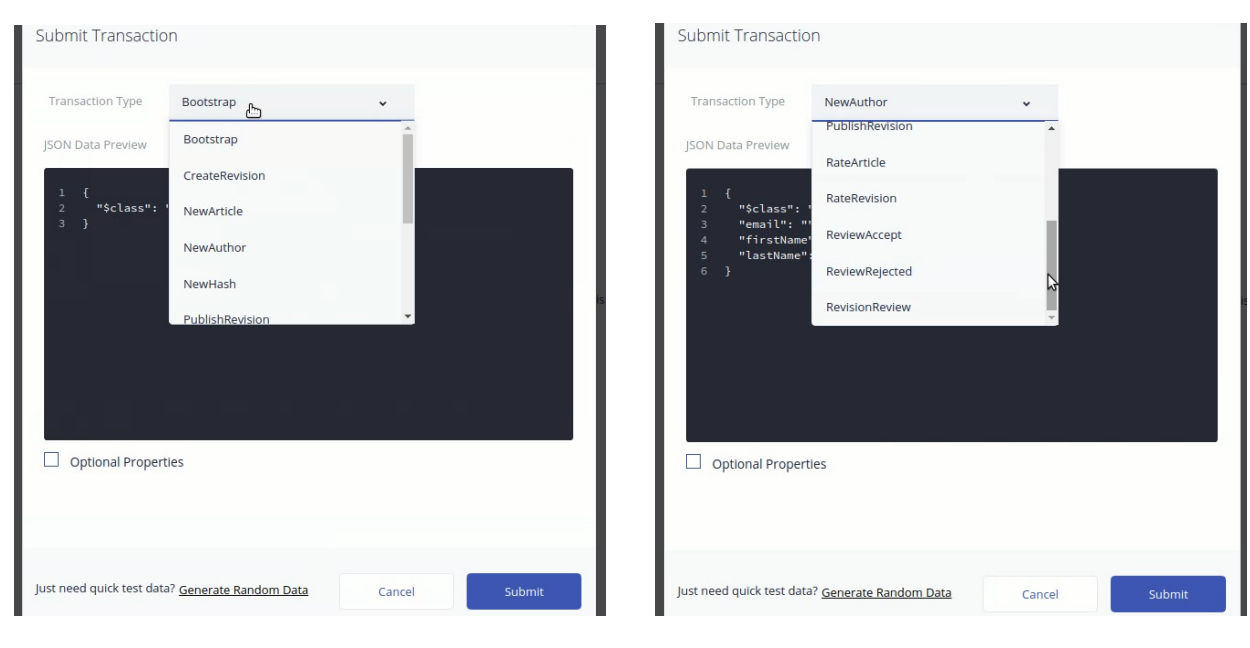

$\begin{aligned} & \text { Figura 8. Funções da } \\ & \text { DASP }\end{aligned}$
$\begin{aligned} & \text { Figura } 9 . \\ & \text { DASP }\end{aligned}$

- RateRevision: É a opção onde se determina a avaliação, seguido por comentários acerca do trabalho;

- ReviewAccept: Determina se o artigo foi aceito, baseado na revisão;

- ReviewRejected: Determina se o artigo foi Recusado, baseado na revisão;

- RevisionReview: Aqui temos a opção de revisão secundária, ou revisão da revisão, onde o próprio DASP, baseado em termos de qualidade da revisão, determina se será necessário ou não uma nova revisão.

A Figura 10 mostra o registro geral de todas as transações realizadas no decorrer do processo, podemos a partir desse registro gerar auditorias e relatórios do que foi feito na aplicação de modo geral.

Em relação aos testes realizados, não realizamos testes de efetivos de avaliação e eficiencia, porém tivemos boas respostas da ferramenta, relacionados ao tempo de interação entre os entes e o uso da rede privada, agregando os mecanismo de segurança da mesma. Entendemos que o futuro das propostas citadas nos trabalhos relacionados será a migração de uma rede pública para as permissionadas, por motivos de segurança na interação da rede entre os entes e o acesso as informações. 


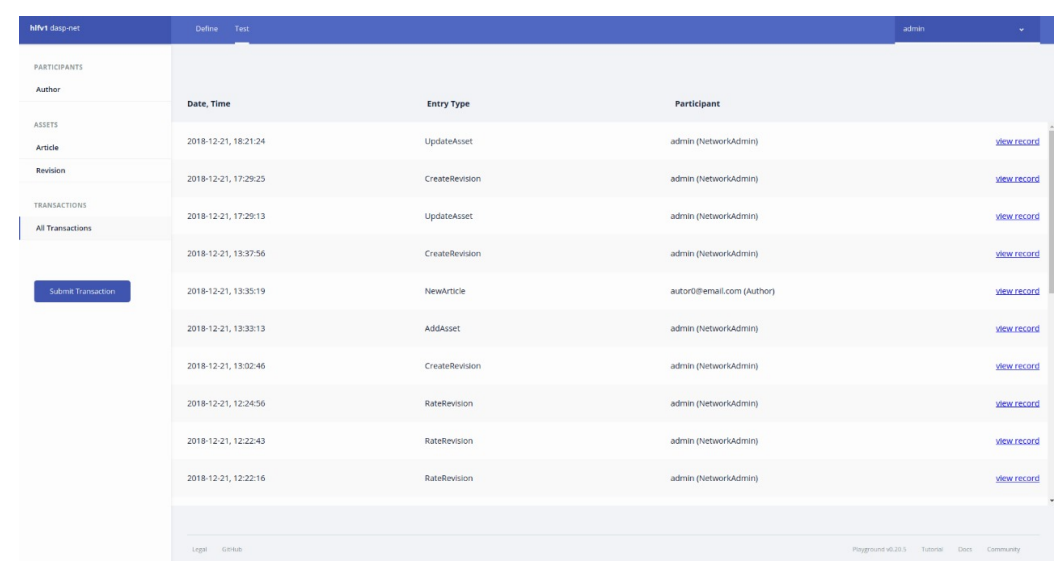

Figura 10. Registro das Transações Realizadas

\section{Considerações Finais e Trabalhos Futuros}

Os processos atuais de submissão, as entidades e nuances envolvidas na revisão por pares, acaba gerando um problema em relação a qualidade e o tempo das revisões, e acima de tudo, a ausência de controle, por parte dos autores, sobre os direitos autorais do seu próprio trabalho.

Nesse sentido a DASP, é proposta para autonomizar o ente editora, responsável pela gestão de todo o processo de revisão e publicação. A ideia por trás dessa alternativa é permitir maior autonomia e confiança entre as entidades que realmente fazem o processo existir, retirando o entidade intermediário (editora), promovendo uma comunicação Peerto-peer( $\mathrm{p} 2 \mathrm{p})$, baseado em regras de consenso entre os membros da comunidade.

Desta forma, umas das principais mudanças seria como o autor, o revisor, o leitor e a comunidade passariam a se relacionar no processo, tendo em vista que todos teriam maior autonomia de opinião no processo, por exemplo, caso o autor se sinta desconfortável com a primeira revisão, ele poderá solicitar uma revisão secundária por parte de outros membros da comunidade. Só esse ponto já é uma iniciativa totalmente diferente comparada as atuais. Essa abordagem acaba dando maior incentivo aos revisores a terem mais atenção aos trabalhos, isso não seria apenas uma mudança de comportamento, mas também de respeito ao pesquisador e o grupo que ele estiver envolvido.

Como trabalhos futuros, iremos implementar um front-end para a aplicação juntamente com funcionalidades mais intuitivas, aperfeiçoar modelos de revisões de acordo com os mecanismos de consenso existentes e desenvolver novas soluções que facilitem o uso de instâncias por parte de usuários que desejem expandir a rede de interações com DASP.

\section{Referências}

Benet, J. (2014). Ipfs-content addressed, versioned, $\mathrm{p} 2 \mathrm{p}$ file system. arXiv preprint arXiv:1407.3561.

Cachin, C. (2016). Architecture of the hyperledger blockchain fabric. In Workshop on Distributed Cryptocurrencies and Consensus Ledgers. 
de Lemos, M. F., Oliveira, P. C., Ruela, L. C., da Silva Santos, M., Slveira, T. C., and de Sousa Reis, J. C. (2013). Aplicabilidade da arquitetura mvc em uma aplicação web (webapps). RE3C-Revista Eletrônica Científica de Ciência da Computação, 8(1).

DEIP, W. (2018). Decentralized research platform. Url: https://deip.world/. Ultimo Acesso: 2018-12-20.

Di Crescenzo, G., Katz, J., Ostrovsky, R., and Smith, A. (2001). Efficient and noninteractive non-malleable commitment. In Pfitzmann, B., editor, Advances in Cryptology - EUROCRYPT 2001, pages 40-59, Berlin, Heidelberg.

DuPont, Q. (2017). Experiments in algorithmic governance: A history and ethnography of "the dao," a failed decentralized autonomous organization. Bitcoin and Beyond: Cryptocurrencies, Blockchains and Global Governance. Routledge.

EurekaPlatform (2018). Eureka, a peer-to-peer scientific data publishing platform, powered by blockchain, to promote open science. https://eurekatoken.io/whitepaper_v3_1.pdf. Ultimo Acesso: 2018-12-21.

Ferraz, E. d. C. and Navas, A. L. G. P. (2016). Publicação de artigos científicos: recomendações práticas para jovens pesquisadores. Associação Brasileira de Editores Científicos.

Frantz, C. K. and Nowostawski, M. (2016). From institutions to code: Towards automated generation of smart contracts. In 2016 IEEE 1st International Workshops on Foundations and Applications of Self* Systems (FAS*W), pages 210-215.

Greve, F., Sampaio, L., ABIJAUDE, J., COUTINHO, A., VALCY, I., and Queiroz, S. (2018). Blockchain e a revolução do consenso sob demanda. Livro de Minicursos do SBRC, 1:1-52.

IEEE, F. (2013). iee faqs. Url: http://www.ieee.org.br/ieee2013/wpcontent/uploads/2013/01/revista_final.pdf/. Ultimo Acesso: 2018-03-26.

Job, I., Trindade, A., and Mattos, A. M. (2009). Processo de revisão pelos pares: Por que são rejeitados os manuscritos submetidos a um periódico científico? Movimento (ESEFID/UFRGS), 15(3):35-55.

Mulligan, A., Hall, L., and Raphael, E. (2012). Peer review in a changing world: An international study measuring the attitudes of researchers. Journal of the American Society for Information Science and Technology, 64(1):132-161.

Partners, N. (2018). Open science network. Url: https://www.osn.global/. Ultimo Acesso: 2018-11-05.

SBC (2015). Sbc. Url: http://seer.ufrgs.br/index.php/reic/about/submissions\#privacyStatement Ultimo Acesso: 2018-05-09.

Shurkin, Joel (2017). Acm history. Url:https://www.acm.org/about-acm/acm-history Ultimo Acesso: 2018-05-09.

Tschorsch, F. and Scheuermann, B. (2016). Bitcoin and beyond: A technical survey on decentralized digital currencies. IEEE Comm. Surveys Tutorials, 18(3):2084-2123.

Zheng, Z., Xie, S., Dai, H.-N., and Wang, H. (2016). Blockchain challenges and opportunities: A survey. Work Pap. 